\title{
Mathematical Analysis of the Effect of Control on the Dynamics of Diabetes Mellitus and Its Complications
}

\author{
Patrick Olabanji Aye \\ Department of Mathematical Sciences, Adekunle Ajasin University, Akungba-Akoko, \\ Ondo State, Nigeria; e-mail: patrick.aye@aaua.edu.ng; ayepatricko@gmail.com
}

\begin{abstract}
Diabetes is a disorder in which the body becomes unable to control the amount of sugar in the blood. The pancreas (beta-cells) is not functioning normally, resulting in a partial or total lack of insulin which is the key to the mechanism converting sugar to energy. In this study, mathematical model for the dynamics of diabetes mellitus and its complications incorporating control is developed and analyzed. Positive lifestyle, which includes abstinence from alcohol, smoking and glutoning, and effective management of diabetes condition are incorporated as controls. The analytical solution of the model equations is obtained using Homotopy Perturbation Method. Numerical simulation of the model solution was done using Maple 18 Mathematical software. The parameters are varied and their effects on the model dynamics are presented graphically. The results showed that the two control measures can effectively be used to reduce the evolution of incidence of diabetes and occurrence of complications of diabetes thereby reducing the rate of morbidity and mortality due to diabetes complications.
\end{abstract}

\section{Introduction}

Diabetes is a metabolic disorder characterized by the inability of the body system to regulate the amount of glucose (special sugar in the body) in the body. This occurs when the insulin secretes by the organ (pancreas) in the beta cell is not sufficient or the body does not utilise the insulin produced effectively. The resulting effect is that the body system cannot function effectively and glucose level in the blood goes beyond normal [37], [36], [34], and [24]. The menace of diabetes and it effect on human, economy and social impact have become a serious problem to the world. International Diabetes Received: January 19, 2021; Accepted: February 28, 2021 2010 Mathematics Subject Classification: 93A30, 92B05, 97M60.

Keywords and phrases: diabetes mellitus, complications, homotopy perturbation method, simulation and mathematical model. 
Federation [27] and World Health Organization [36], reported that $2.9-8.4$ million deaths worldwide are attributable to diabetes. An undiagnosed or uncontrolled diabetes leads to complications and complications lead to deaths. Rising cases of cardiovascular disease, renal failure, blindness, cognitive and psychiatric illness and infections are attributable to diabetes. Diabetes causes loss of sensory and motor function, uneven blood circulation in the feet and hand, and poor wound healing.

Diabetes coexists with infections. This can be seen in diabetes and tuberculosis and group B Streptococci [11], [28]. The relationship between diabetes and tuberculosis slow down recovery rate and increase risk of tuberculosis relapse, resistance and death [28]. Diabetes also coexists with Human Immunodeficiency Virus (HIV) thereby causing early death [4]. The number of deaths attributable to diabetes yearly worldwide is alarming. Recently, there has been an increase in life expectancy around the world. Death rate caused by other leading non-communicable diseases such as Cancer, Cardiovascular disease and Stroke have been decreasing but that of diabetes is rising [5]. According to WHO, 2.8 percent of all deaths worldwide in 2010 are attributable to diabetes excluding deaths caused by cardiovascular disease and chronic kidney disease. 21 percent of coronary heart disease and 13 percent of stroke mortality worldwide are caused by diabetes [9]. Also, International Diabetes Federation revealed that 5 million adult deaths yearly, or 8.4 percent of all deaths worldwide are attributable to diabetes [26], [32].

This paper expanded the scope of the dynamics of diabetes mellitus by adding three classes and incorporates control measures. The classes added are healthy class, susceptible class and diabetics with complications undergoing treatment class while the control measures are positive lifestyle and effective management of diabetes conditions. In section two, we described the proposed model and its parameters and obtained the analytical solution of the model equations. In section three, we carried out numerical simulation of the results obtained in section two and presents the graphical profile of the system responses. In section four, we discussed our results.

\subsection{Homotopy perturbation method (HPM)}

The method of Homotopy perturbation for solving problems of nonlinear equation was first introduced by He [16], [17] and subsequently, it was improved by [18], [19], [20], [21] and [22]. The method was successfully applied to solve problems made up of nonlinear and linear functional equations. In recent years, scientists, technologists and engineers have applied homotopy perturbation method to deform problem of nonlinear and linear differential equations that seem to be difficult into simpler one that is easier to 
solve. The method is a combination of homotopy method in topology and traditional perturbation method [23]. The method of homotopy perturbation have provides a very convenient approach for obtaining analytical or approximate solution of series of problems in various field.

Many researchers have successfully applied the method to physical problems such as non-linear wave equations, bifurcation, asymptotology, SIR infection disease model [1], equation of Schrodinger [6], diabetes model [15], heat radiation equation and reactiondiffusion equation [13], [14], oscillators with discontinuities [20], [21], Sine-Gordon and Klein-Gordon equations [29], [8].

\subsection{Fundamental of homotopy perturbation method}

The basic illustration of the method according to [17], using non-linear differential equation is stated as follows:

$$
D(u)-k(x)=0, \quad x \in \Lambda
$$

with boundary condition of:

$$
E\left(u, \frac{\partial u}{\partial n}\right)=0, \quad x \in \Pi
$$

$D$ denotes general differential operator, the boundary operator is $E$, the known analytical function is $k(x)$ and $\Pi$ denotes domain boundary of $\Lambda$. The operator $D$ can be divided into two parts of $G$ and $H$, the linear part is $G$, while $H$ is a non-linear one. Equation (1.1) can be rewritten as follows:

$$
G(u)+H(u)-k(x)=0, \quad x \in \Lambda .
$$

The stated HPM equation is as follows:

$$
Q(w, r)=(1-r)\left[G(w)-G\left(u_{0}\right)\right]+r[G(w)+H(w)-k(x)]=0 .
$$

The embedding parameter is $r \in[0,1]$ in equation (1.4) and the first assumption that meets the boundary condition is $\mu_{0}$. The assumed solution of equation (1.4) can be expressed in power series as follows:

$$
w=w_{0}+r w_{1}+r^{2} w_{2}+\cdots .
$$

The correct solution of (1.1) is obtained by setting $r=1$

$$
u=\lim _{p \rightarrow 1} w=w_{0}+w_{1}+w_{2}+\cdots .
$$


In most cases, the series (1.5) converges. The convergent rate depends on the nonlinear operator $D(w)$ [17].

\section{Materials and Methods}

\subsection{Model formulation}

The study considered the model proposed by [15] with some modifications and incorporate two control measures. The control measures are positive lifestyle and effective management of diabetes conditions. Based on their health status, the model population are classified into five classes. They are healthy class $H(t)$, susceptible class $S(t)$, diabetic without complications class $D(t)$, diabetic with complications class $C(t)$, and diabetic with complications that undergo treatment class $T(t)$. The assumption of the model was that the healthy individual will give birth to a healthy children that will be born into healthy compartment while parent who is diabetic or have history of diabetes will give birth to children with genetic factors that will be born into susceptible compartment. The proportion of children born into healthy compartment is denoted by $\theta$ while proportion of children that are born into susceptible compartment is denoted by $1-\theta$. The control parameters are $\phi_{1}, \phi_{2}$. $\phi_{1}$ is a measure of positive lifestyle in the susceptible class, such that $0 \leq \phi_{1} \leq 1 . \phi_{1}=0$ indicate negative lifestyle and $\phi_{1}=1$ indicate positive lifestyle. $\phi_{2}$ is a measure of effective management of diabetes condition in the compartment of diabetics without complications, such that $0 \leq \phi_{2} \leq 1 . \phi_{2}=0$ indicate ineffective management of diabetes condition and $\phi_{2}=1$ indicate effective management of diabetes condition.

Table 2.1. Definition of variables of the model.

\begin{tabular}{|l|l|l|}
\hline $\mathbf{S} / \mathbf{N}$ & Variables & Description \\
\hline 1 & $H(t)$ & Healthy class \\
\hline 2 & $S(t)$ & Susceptible class \\
\hline 3 & $D(t)$ & Diabetics without complications class \\
\hline 4 & $C(t)$ & Diabetics with complications class \\
\hline 5 & $T(t)$ & Diabetics with complications undergoing treatment class \\
\hline 6 & $N(t)$ & Total population \\
\hline
\end{tabular}


Table 2.2. Definition of parameters of the model.

\begin{tabular}{|l|l|l|}
\hline S/N & Parameters & Description \\
\hline 1 & $\alpha$ & Probability rate of incidence of diabetes \\
\hline 2 & $\beta$ & Birth rate \\
\hline 3 & $\mu$ & Natural mortality rate \\
\hline 4 & $\tau$ & Rate at which healthy individual become susceptible \\
\hline 5 & $\sigma$ & Rate at which susceptible individual become healthy \\
\hline 6 & $\lambda$ & Rate at which $D(t)$ develop a complications \\
\hline 7 & $\gamma$ & Rate at which $C(t)$ are treated \\
\hline 8 & $\omega$ & Rate at which $C(t)$ after treatment return to $D(t)$ \\
\hline 9 & $\delta$ & Mortality rate due to complications \\
\hline 10 & $\theta$ & Proportion of children born into the healthy class \\
\hline 11 & $\phi_{1}$ & Measure of positive lifestyle in $S(t)$ class \\
\hline 12 & $\phi_{2}$ & $\begin{array}{l}\text { Measure of effective management of diabetes condition in } D(t) \\
\text { class }\end{array}$ \\
\hline 13 & $1-\theta$ & Proportion of children born into the susceptible class \\
\hline
\end{tabular}

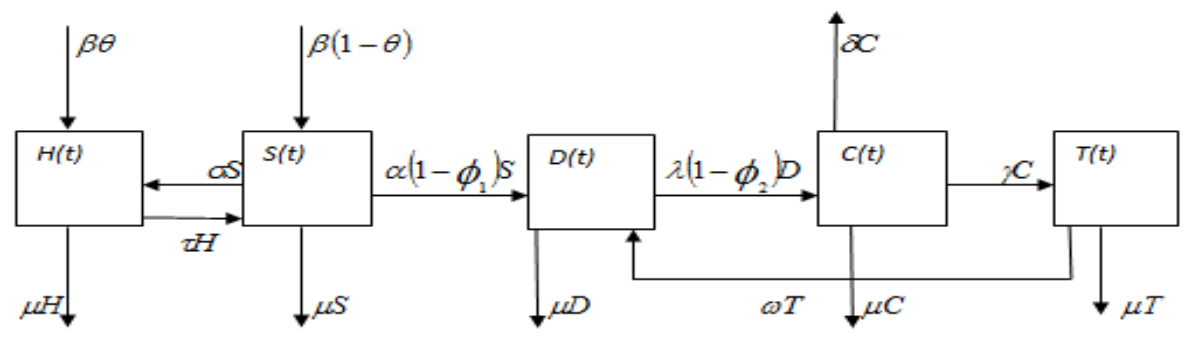

Figure 2.1. Schematic diagram of the model.

\subsection{The model equations}

The model equations are stated as follows in (2.1) to (2.5)

$$
\frac{d H(t)}{d t}=\sigma S(t)-\mu H(t)-\tau H(t)+\beta \theta,
$$




$$
\begin{aligned}
& \frac{d S(t)}{d t}=\beta(1-\theta)-\mu S(t)+\tau H(t)-\alpha\left(1-\phi_{1}\right) S(t)-\sigma S(t), \\
& \frac{d D(t)}{d t}=\alpha\left(1-\phi_{1}\right) S(t)+\omega T(t)-\lambda\left(1-\phi_{2}\right) D(t)-\mu D(t) \\
& \frac{d C(t)}{d t}=\lambda\left(1-\phi_{2}\right) D(t)-\gamma C(t)-\delta C(t)-\mu C(t) \\
& \frac{d T(t)}{d t}=\gamma C(t)-\omega T(t)-\mu T(t) .
\end{aligned}
$$

The initial values conditions are $H(o)=H_{o}, S(o)=S_{o}, D(o)=D_{o}, C(o)=C_{o}$ and $T(o)=T_{o}$.

2.3. Analytical solution of the model equations by homotopy perturbation method (HPM)

Consider the systems:

$$
\begin{aligned}
& \frac{d H}{d t}=\beta \theta-(\mu+\tau) H+\sigma S \\
& \frac{d S}{d t}=\beta(1-\theta)+\tau H-\mu S-\sigma S-\alpha\left(1-\phi_{1}\right) S, \\
& \frac{d D}{d t}=\alpha\left(1-\phi_{1}\right) S-\mu D-\lambda\left(1-\phi_{2}\right) D+\omega T \\
& \frac{d C}{d t}=\lambda\left(1-\phi_{2}\right) D-(\mu+\delta+\gamma) C \\
& \frac{d T}{d t}=\gamma C-(\mu+\omega) T .
\end{aligned}
$$

Equations (2.1) to (2.5) can be written as

$$
\begin{aligned}
& \frac{d H}{d t}+Q_{1} H-\sigma S-\beta \theta=0, \\
& \frac{d S}{d t}-\tau H+Q_{2} S-\beta(1-\theta)=0,
\end{aligned}
$$




$$
\begin{aligned}
& \frac{d D}{d t}-\alpha\left(1-\phi_{1}\right) S+Q_{3} D-\omega T=0, \\
& \frac{d C}{d t}-\lambda\left(1-\phi_{2}\right) D+Q_{4} C=0, \\
& \frac{d T}{d t}-\gamma C+Q_{5} T=0,
\end{aligned}
$$

where

$$
\begin{aligned}
& Q_{1}=\mu+\tau, \\
& Q_{2}=\mu+\sigma+\alpha-\alpha \phi_{1}, \\
& Q_{3}=\mu+\lambda-\lambda \phi_{2}, \\
& Q_{4}=\mu+\delta+\gamma, \\
& Q_{5}=\mu+\omega,
\end{aligned}
$$

with initial conditions $H(0)=H_{0}, S(0)=S_{0}, D(0)=D_{0}, C(0)=C_{0}$ and $T(0)=T_{0}$.

Let

$$
\begin{aligned}
& H(t)=b_{0}+p b_{1}+p^{2} b_{2}+\cdots \\
& S(t)=e_{0}+p e_{1}+p^{2} e_{2}+\cdots \\
& D(t)=f_{0}+p f_{1}+p^{2} f_{2}+\cdots \\
& C(t)=g_{0}+p g_{1}+p^{2} g_{2}+\cdots \\
& T(t)=j_{0}+p j_{1}+p^{2} j_{2}+\cdots
\end{aligned}
$$

Applying HPM to (2.11) gives

$$
(1-p) \frac{d H}{d t}+p\left[\frac{d H}{d t}+Q_{1} H-\sigma S-\beta \theta\right]=0 .
$$

Substituting (2.21) to (2.25) into (2.26a) and simplify gives 


$$
\begin{gathered}
(1-p)\left[b_{0}^{\prime}+p b_{1}^{\prime}+p^{2} m_{2}^{\prime}+\cdots\right]+p\left[\begin{array}{c}
b_{0}^{\prime}+p b_{1}^{\prime}+p^{2} b_{2}^{\prime}+\cdots \\
+Q_{1}\left(b_{0}+p b_{1}+p^{2} b_{2}+\cdots\right) \\
-\sigma\left(e_{0}+p e_{1}+p^{2} e_{2}+\cdots\right)-\beta \theta
\end{array}\right]=0 \\
b_{0}^{\prime}+p b_{1}^{\prime}+p^{2} b_{2}^{\prime}+\cdots+p Q_{1}\left(b_{0}+p b_{1}+p^{2} b_{2}+\cdots\right) \\
-p \sigma\left(e_{0}+p e_{1}+p^{2} e_{2}+\cdots\right)-p \beta \theta=0
\end{gathered}
$$

Collecting the coefficients of power of $p$ in (2.26b) gives

$$
\left.\begin{array}{l}
p^{0}: b_{0}^{\prime}=0 \\
p^{1}: b_{1}^{\prime}+Q_{1} b_{0}-\sigma e_{0}-\beta \theta=0 \\
p^{2}: b_{2}^{\prime}+Q_{1} b_{1}-\sigma e_{1}=0
\end{array}\right\} .
$$

Applying HPM to (2.12) gives

$$
(1-p) \frac{d S}{d t}+p\left[\frac{d S}{d t}-\tau H+Q_{2} S+\beta \theta-\beta\right]=0 .
$$

Substituting (2.21) to (2.25) into (2.28) and simplify gives

$$
\begin{gathered}
(1-p)\left[e_{0}^{\prime}+p e_{1}^{\prime}+p^{2} e_{2}^{\prime}+\cdots\right]+p\left[\begin{array}{c}
e_{0}^{\prime}+p e_{1}^{\prime}+p^{2} e_{2}^{\prime}+\cdots \\
-\tau\left(b_{0}+p b_{1}+p^{2} b_{2}+\cdots\right) \\
+Q_{2}\left(e_{0}+p e_{1}+p^{2} e_{2}+\cdots\right) \\
+\beta \theta-\beta
\end{array}\right]=0, \\
e_{0}^{\prime}+p e_{1}^{\prime}+p^{2} e_{2}^{\prime}+\cdots-p \tau\left(b_{0}+p b_{1}+p^{2} b_{2}+\cdots\right) \\
+p Q_{2}\left(e_{0}+p e_{1}+p^{2} e_{2}+\cdots\right)+p \beta \theta-p \beta=0 .
\end{gathered}
$$

Collecting the coefficients of powers of $p$ in (2.29) gives

$$
\left.\begin{array}{l}
p^{0}: e_{0}^{\prime}=0 \\
p^{1}: e_{1}^{\prime}-\tau b_{0}+Q_{2} e_{0}+\beta \theta-\beta=0 \\
p^{2}: e_{2}^{\prime}-\tau b_{1}+Q_{2} e_{1}=0
\end{array}\right\} .
$$

Applying HPM to (2.13) gives

$$
(1-p) \frac{d D}{d t}+p\left[\frac{d D}{d t}-\alpha S+\alpha \phi_{1} S+Q_{3} D-\omega T\right]=0 .
$$


Substituting (2.21) to (2.25) into (2.31) and simplify gives

$$
(1-p)\left[f_{0}^{\prime}+p f_{1}^{\prime}+p^{2} f_{2}^{\prime}+\cdots\right]+p\left[\begin{array}{l}
f_{0}^{\prime}+p f_{1}^{\prime}+p^{2} f_{2}^{\prime}+\cdots \\
-\alpha\left(e_{0}+p e_{1}+p^{2} e_{2}+\cdots\right) \\
+\alpha \phi_{1}\left(e_{0}+p e_{1}+p^{2} e_{2}+\cdots\right) \\
+Q_{3}\left(f_{0}+p f_{1}+p^{2} f_{2}+\cdots\right) \\
-\omega\left(j_{0}+p j_{1}+p^{2} j_{2}+\cdots\right)
\end{array}\right]=0,
$$

Collecting the coefficients of the powers of $p$ in (2.32) gives

$$
\left.\begin{array}{l}
p^{0}: f_{0}^{\prime}=0 \\
p^{1}: f_{1}^{\prime}-\alpha e_{0}+\alpha \phi_{1} e_{0}+Q_{3} f_{0}-\omega j_{0}=0 \\
p^{2}: f_{2}^{\prime}-\alpha e_{1}+\alpha \phi_{1} e_{1}+Q_{3} f_{1}-\omega j_{1}=0
\end{array}\right\} .
$$

Applying HPM to (2.14) gives

$$
(1-p) \frac{d C}{d t}+p\left[\frac{d C}{d t}-\lambda D+\lambda \phi_{2} D+Q_{4} C\right]=0 .
$$

Substituting (2.21) to (2.25) into (2.34) and simplify gives

$$
\begin{gathered}
(1-p)\left[g_{0}^{\prime}+p g_{1}^{\prime}+p^{2} g_{2}^{\prime}+\cdots\right]+p\left[\begin{array}{c}
g_{0}^{\prime}+p g_{1}^{\prime}+p^{2} g_{2}^{\prime}+\cdots \\
-\lambda\left(f_{0}+p f_{1}+p^{2} f_{2}+\cdots\right) \\
+\lambda \phi_{2}\left(f_{0}+p f_{1}+p^{2} f_{2}+\cdots\right) \\
+Q_{4}\left(g_{0}+p g_{1}+p^{2} g_{2}+\cdots\right)
\end{array}\right]=0, \\
g_{0}^{\prime}+p g_{1}^{\prime}+p^{2} g_{2}^{\prime}+\cdots-p \lambda\left(f_{0}+p f_{1}+p^{2} f_{2}+\cdots\right) \\
+p \lambda \phi_{2}\left(f_{0}+p f_{1}+p^{2} f_{2}+\cdots\right)+p Q_{4}\left(g_{0}+p g_{1}+p^{2} g_{2}+\cdots\right)=0 .
\end{gathered}
$$

Collecting the coefficients of the powers of $p$ in (2.35) gives

$$
\left.\begin{array}{l}
p^{0}: g_{0}^{\prime}=0 \\
p^{1}: g_{1}^{\prime}-\lambda f_{0}+\lambda \phi_{2} f_{0}+Q_{4} g_{0}=0 \\
p^{2}: g_{2}^{\prime}-\lambda f_{1}+\lambda \phi_{2} f_{1}+Q_{4} g_{1}=0
\end{array}\right\} .
$$


Applying HPM to (2.15) gives

$$
(1-p) \frac{d T}{d t}+p\left[\frac{d T}{d t}-\gamma D+Q_{3} T\right]=0 .
$$

Substituting (2.21) to (2.25) into (2.37) and simplify gives

$$
\begin{gathered}
(1-p)\left[j_{0}^{\prime}+p j_{1}^{\prime}+p^{2} j_{2}^{\prime}+\cdots\right]+p\left[\begin{array}{c}
j_{0}^{\prime}+p j_{1}^{\prime}+p^{2} j_{2}^{\prime}+\cdots \\
-\gamma\left(g_{0}+p g_{1}+p^{2} g_{2}+\cdots\right) \\
+Q_{5}\left(j_{0}+p j_{1}+p^{2} j_{2}+\cdots\right)
\end{array}\right]=0 \\
j_{0}^{\prime}+p j_{1}^{\prime}+p^{2} j_{2}^{\prime}+\cdots-p \gamma\left(g_{0}+p g_{1}+p^{2} g_{2}+\cdots\right) \\
+p k_{5}\left(j_{0}+p j_{1}+p^{2} j_{2}+\cdots\right)=0 .
\end{gathered}
$$

Collecting the coefficients of powers of $p$ in (2.38) gives

$$
\left.\begin{array}{l}
p^{0}: j_{0}^{\prime}=0 \\
p^{1}: j_{1}^{\prime}-\gamma g_{0}+Q_{5} j_{0}=0 \\
p^{2}: j_{2}^{\prime}-\gamma g_{1}+Q_{5} j_{1}=0
\end{array}\right\} .
$$

Solving the first equations of (2.27), (2.30), (2.33), (2.36) and (2.39) gives

$$
\left.\begin{array}{l}
b_{0}=H_{0} \\
e_{0}=S_{0} \\
f_{0}=D_{0} \\
g_{0}=C_{0} \\
j_{0}=T_{0}
\end{array}\right\} .
$$

Substituting (2.40) into second equations of (2.27), (2.30), (2.33), (2.36) and (2.39) gives

$$
\left.\begin{array}{l}
b_{1}^{\prime}+Q_{1} H_{0}-\sigma S_{0}-\beta \theta=0 \\
e_{1}^{\prime}-\tau H_{0}+Q_{2} S_{0}+\beta \theta-\beta=0 \\
f_{1}^{\prime}-\alpha S_{0}+\alpha \phi_{1} S_{0}+Q_{3} D_{0}-\omega T_{0}=0 \\
g_{1}^{\prime}-\lambda D_{0}+\lambda \phi_{2} D_{0}+Q_{4} C_{0}=0 \\
j_{1}-\gamma C_{0}+Q_{3} T_{0}=0
\end{array}\right\} .
$$

Solving (2.41) gives 


$$
\left.\begin{array}{l}
b_{1}=\left(\beta \theta-Q_{1} H_{0}+\sigma S_{0}\right) t \\
e_{1}=\left(\tau H_{0}-Q_{2} S_{0}-\beta \theta+\beta\right) t \\
f_{1}=\left(\alpha S_{0}-\alpha \phi_{1} S_{0}-Q_{3} D_{0}+\omega T_{0}\right) t \\
g_{1}=\left(\lambda D_{0}-\lambda \phi_{2} D_{0}-Q_{4} C_{0}\right) t \\
j_{1}=\left(\gamma C_{0}-Q_{3} T_{0}\right) t
\end{array}\right\} .
$$

Substituting (2.42) into the third equations of (2.27), (2.30), (2.33), (2.36) and (2.39) gives

$$
\begin{aligned}
& \int b_{2}^{\prime} d t=-Q_{1} \int\left(\beta \theta-Q_{1} H_{0}+\sigma S_{0}\right) t d t+\sigma \int\left(\tau H_{0}-Q_{2} S_{0}-\beta \theta+\beta\right) t d t \\
& b_{2}=\left[\sigma\left(\tau H_{0}-Q_{2} S_{0}-\beta \theta+\beta\right)-Q_{1}\left(\beta \theta-Q_{1} H_{0}+\sigma S_{0}\right)\right] \frac{t^{2}}{2}+\cdots \\
& \int e_{2}^{\prime} d t=\tau \int\left(\beta \theta-Q_{1} H_{0}+\sigma S_{0}\right) t d t-Q_{2} \int\left(\tau H_{0}-Q_{2} S_{0}-\beta \theta+\beta\right) t d t \\
& e_{2}=\left[\tau\left(\beta \theta-Q_{1} H_{0}+\sigma S_{0}\right)-Q_{2}\left(\tau H_{0}-Q_{2} S_{0}-\beta \theta+\beta\right)\right] \frac{t^{2}}{2}+\cdots \\
& \int f_{2}^{\prime} d t=\alpha \int\left(\tau H_{0}-Q_{2} S_{0}-\beta \theta+\beta\right) t d t-Q_{3} \int\left(\alpha S_{0}-Q_{3} D_{0}+\omega T_{0}\right) t d t \\
& +\omega \int\left(\gamma C_{0}-Q_{5} T_{0}\right) t d t \\
& f_{2}=\left[\begin{array}{l}
\sigma\left(\tau H_{0}-Q_{2} S_{0}-\beta \theta+\beta\right)-\alpha \phi_{1}\left(\tau H_{0}-Q_{2} S_{0}-\beta \theta+\beta\right) \\
-Q_{3}\left(\alpha S_{0}-\alpha \phi_{1} S_{0}-Q_{3} D_{0}+\omega T_{0}\right)+\omega\left(\gamma C_{0}-Q_{5} T_{0}\right)
\end{array}\right] \frac{t^{2}}{2}+\cdots \\
& \int g_{2}^{\prime} d t=\lambda \int\left(\alpha S_{0}-Q_{3} D_{0}+\omega T_{0}\right) t d t-\lambda \phi \int\left(\alpha S_{0}-Q_{3} D_{0}+\omega T_{0}\right) t d t \\
& -Q_{4} \int\left(\lambda D_{0}-\lambda \phi_{2} D_{0}-Q_{4} C_{0}\right) t d t \\
& g_{2}=\left[\begin{array}{c}
\lambda\left(\alpha S_{0}-\alpha \phi_{1} S_{0}-Q_{3} D_{0}+\omega T_{0}\right) \\
-\lambda \phi_{2}\left(\alpha S_{0}-\alpha \phi_{1} S_{0}-Q_{3} D_{0}+\omega T_{0}\right) \\
-Q_{4}\left(\lambda D_{0}-\lambda \phi_{2} D_{0}-Q_{4} C_{0}\right)
\end{array}\right] \frac{t^{2}}{2}+\cdots \\
& \int j_{2}^{\prime} d t=\gamma \int\left(\lambda D_{0}-\lambda \phi_{2} D_{0}-Q_{4} C_{0}\right) t d t-Q_{5} \int\left(\gamma C_{0}-Q_{5} T_{0}\right) t d t \\
& j_{2}=\left[\gamma\left(\lambda D_{0}-\lambda \phi_{2} D_{0}-Q_{4} C_{0}\right)-Q_{5}\left(\gamma C_{0}-Q_{5} T_{0}\right)\right] \frac{t^{2}}{2}+\cdots
\end{aligned}
$$




$$
\left.\begin{array}{rl}
b_{2}= & {\left[\sigma\left(\tau H_{0}-Q_{2} S_{0}-\beta \theta+\beta\right)-Q_{1}\left(\beta \theta-Q_{1} H_{0}+\sigma S_{0}\right)\right] \frac{t^{2}}{2}+\cdots} \\
e_{2}= & {\left[\tau\left(\beta \theta-Q_{1} H_{0}+\sigma S_{0}\right)-Q_{2}\left(\tau H_{0}-Q_{2} S_{0}-\beta \theta+\beta\right)\right] \frac{t^{2}}{2}+\cdots} \\
f_{2}= & {\left[\begin{array}{l}
\sigma\left(\tau H_{0}-Q_{2} S_{0}-\beta \theta+\beta\right)-\alpha \phi_{1}\left(\tau H_{0}-Q_{2} S_{0}-\beta \theta+\beta\right) \\
-Q_{3}\left(\alpha S_{0}-\alpha \phi_{1} S_{0}-Q_{3} D_{0}+\omega T_{0}\right)+\omega\left(\gamma C_{0}-Q_{5} T_{0}\right)
\end{array}\right] \frac{t^{2}}{2}+\cdots} \\
g_{2}= & {\left[\begin{array}{c}
\lambda\left(\alpha S_{0}-\alpha \phi_{1} S_{0}-Q_{3} D_{0}+\omega T_{0}\right) \\
-\lambda \phi_{2}\left(\alpha S_{0}-\alpha \phi_{1} S_{0}-Q_{3} D_{0}+\omega T_{0}\right) \\
-Q_{4}\left(\lambda D_{0}-\lambda \phi_{2} D_{0}-Q_{4} C_{0}\right)
\end{array}\right] \cdot \frac{t^{2}}{2}+\cdots}
\end{array}\right] .
$$

Substituting (2.40), (2.42) and (2.48) into (2.21) gives

$$
H(t)=H_{0}+p\left[\left(\beta \theta-Q_{1} H_{0}+\sigma S_{0}\right) t\right]+p^{2}\left[\begin{array}{c}
\sigma\left(\tau H_{0}-Q_{2} S_{0}-\beta \theta+\beta\right) \\
-Q_{1}\left(\beta \theta-Q_{1} H_{0}+\sigma S_{0}\right)
\end{array}\right] \frac{t^{2}}{2}+\cdots .
$$

Setting $p=1$ of (2.49) becomes

$$
H(t)=\lim _{p \rightarrow 1} b=b_{0}+b_{1}+b_{2}
$$

Hence,

$$
H(t)=H_{0}+\left(\beta \theta-Q_{1} H_{0}+\sigma S_{0}\right) t+\left[\begin{array}{c}
\sigma\left(\tau H_{0}-Q_{2} S_{0}-\beta \theta+\beta\right) \\
-Q_{1}\left(\beta \theta-Q_{1} H_{0}+\sigma S_{0}\right)
\end{array}\right] \frac{t^{2}}{2}+\cdots .
$$

Substituting (2.40), (2.42) and (2.48) into (2.22) gives

$$
S(t)=S_{0}+p\left[\left(\tau H_{0}-Q_{2} S_{0}-\beta \theta+\beta\right) t\right]+p^{2}\left[\begin{array}{c}
\tau\left(\beta \theta-Q_{1} H_{0}+\sigma S_{0}\right) \\
-Q_{2}\left(\tau H_{0}-Q_{2} S_{0}-\beta \theta+\beta\right)
\end{array}\right] \frac{t^{2}}{2}+\cdots .
$$

Setting $p=1$ of (2.52) becomes

$$
S(t)=\lim _{p \rightarrow 1} e=e_{0}+e_{1}+e_{2} .
$$

Hence,

$$
S(t)=S_{0}+\left(\tau H_{0}-Q_{2} S_{0}-\beta \theta+\beta\right) t+\left[\begin{array}{c}
\tau\left(\beta \theta-Q_{1} H_{0}+\sigma S_{0}\right) \\
-Q_{2}\left(\tau H_{0}-Q_{2} S_{0}-\beta \theta+\beta\right)
\end{array}\right] \frac{t^{2}}{2}+\cdots .
$$


Substituting (2.40), (2.42) and (2.48) into (2.23) gives

$$
\begin{aligned}
& D(t)=D_{0}+p\left[\left(\alpha S_{0}-\alpha \phi_{1} S_{0}-D_{3} D_{0}+\omega T_{0}\right) t\right] \\
& +p^{2}\left[\begin{array}{c}
\alpha\left(\tau H_{0}-Q_{2} S_{0}-\beta \theta+\beta\right) \\
-\alpha \phi_{1}\left(\tau H_{0}-Q_{2} S_{0}-\beta \theta+\beta\right) \\
-Q_{3}\left(\alpha S_{0}-\alpha \phi_{1} S_{0}-Q_{3} D_{0}+\omega T_{0}\right) \\
+\omega\left(\gamma C_{0}-Q_{5} T_{0}\right)
\end{array}\right] \frac{t^{2}}{2}+\cdots .
\end{aligned}
$$

Setting $p=1$ of (2.55) becomes

$$
D(t)=\lim _{p \rightarrow 1} f=f_{0}+f_{1}+f_{2} .
$$

Hence,

$$
\begin{aligned}
D(t)=D_{0} & +\left(\alpha S_{0}-\alpha \phi_{1} S_{0}-Q_{3} D_{0}+\omega T_{0}\right) t \\
& +\left[\begin{array}{c}
\alpha\left(\tau H_{0}-Q_{2} S_{0}-\beta \theta+\beta\right) \\
-\alpha \phi_{1}\left(\tau H_{0}-Q_{2} S_{0}-\beta \theta+\beta\right) \\
-Q_{3}\left(\alpha S_{0}-\alpha \phi_{1} S_{0}-Q_{3} D_{0}+\omega T_{0}\right) \\
+\omega\left(\gamma C_{0}-Q_{5} T_{0}\right)
\end{array}\right] \frac{t^{2}}{2}+\cdots
\end{aligned}
$$

Substituting (2.40), (2.42) and (2.48) into (2.24) gives

$$
\begin{aligned}
C(t)=C_{0}+p\left[\left(\lambda D_{0}-\lambda \phi_{2} D_{0}-Q_{4} C_{0}\right) t\right] \\
+p^{2}\left[\begin{array}{c}
\lambda\left(\alpha S_{0}-\alpha \phi_{1} S_{0}-Q_{3} D_{0}+\omega T_{0}\right) \\
-\lambda \phi_{2}\left(\alpha S_{0}-\alpha \phi_{1} S_{0}-Q_{3} D_{0}+\omega T_{0}\right) \\
-Q_{4}\left(\lambda D_{0}-\lambda \phi_{2} D_{0}-Q_{4} C_{0}\right)
\end{array}\right] \frac{t^{2}}{2}+\cdots
\end{aligned}
$$

Setting $p=1$ of (2.58) becomes

$$
C(t)=\lim _{p \rightarrow 1} g=g_{0}+g_{1}+g_{2}
$$

Hence,

$$
\begin{aligned}
C(t)=C_{0}+\left(\lambda D_{0}-\lambda \phi_{2} D_{0}-k_{4} C_{0}\right) t \\
+\left[\begin{array}{c}
\lambda\left(\alpha S_{0}-\alpha \phi_{1} S_{0}-Q_{3} D_{0}+\omega T_{0}\right) \\
-\lambda \phi_{2}\left(\alpha S_{0}-\alpha \phi_{1} S_{0}-Q_{3} D_{0}+\omega T_{0}\right) \\
-Q_{4}\left(\lambda D_{0}-\lambda \phi_{2} D_{0}-Q_{4} C_{0}\right)
\end{array}\right] \frac{t^{2}}{2}+\cdots .
\end{aligned}
$$


Substituting (2.40), (2.42) and (2.48) into (2.25) gives

$$
T(t)=T_{0}+p\left[\left(\gamma C_{0}-Q_{5} T_{0}\right) t\right]+p^{2}\left[\begin{array}{c}
\gamma\left(\lambda D_{0}-\lambda \phi_{2} D_{0}-Q_{4} C_{0}\right) \\
-Q_{5}\left(\gamma C_{0}-Q_{5} T_{0}\right)
\end{array}\right] \frac{t^{2}}{2}+\cdots
$$

Setting $p=1$ of (2.61) becomes

$$
T(t)=\lim _{p \rightarrow 1} j=j_{0}+j_{1}+j_{2}
$$

Hence,

$$
T(t)=T_{0}+\left(\gamma C_{0}-Q_{5} T_{0}\right) t+\left[\begin{array}{c}
\gamma\left(\lambda D_{0}-\lambda \phi_{2} D_{0}-Q_{4} C_{0}\right) \\
-Q_{5}\left(\gamma C_{0}-k_{5} T_{0}\right)
\end{array}\right] \frac{t^{2}}{2}+\cdots .
$$

Equations (2.51), (2.54), (2.57), (2.60) and (2.63) are the solutions of the system of equations (2.1) to (2.5).

$$
\begin{aligned}
& H(t)=H_{0}+\left(\beta \theta-Q_{1} H_{0}+\sigma S_{0}\right) t+\left[\begin{array}{c}
\sigma\left(\tau H_{0}-Q_{2} S_{0}-\beta \theta+\beta\right) \\
-Q_{1}\left(\beta \theta-Q_{1} H_{0}+\sigma S_{0}\right)
\end{array}\right] \frac{t^{2}}{2}+\cdots \\
& S(t)=S_{0}+\left(\tau H_{0}-Q_{2} S_{0}-\beta \theta+\beta\right) t+\left[\begin{array}{c}
\tau\left(\beta \theta-Q_{1} H_{0}+\sigma S_{0}\right) \\
-Q_{2}\left(\tau H_{0}-Q_{2} S_{0}-\beta \theta+\beta\right)
\end{array}\right] \frac{t^{2}}{2}+\cdots \\
& D(t)=D_{0}+\left(\alpha S_{0}-\alpha \phi_{1} S_{0}-Q_{3} D_{0}+\omega T_{0}\right) t \\
& +\left[\begin{array}{c}
\alpha\left(\tau H_{0}-Q_{2} S_{0}-\beta \theta+\beta\right) \\
-\alpha \phi_{1}\left(\tau H_{0}-Q_{2} S_{0}-\beta \theta+\beta\right) \\
-Q_{3}\left(\alpha S_{0}-\alpha \phi_{1} S_{0}-Q_{3} D_{0}+\omega T_{0}\right) \\
+\omega\left(\gamma C_{0}-Q_{5} T_{0}\right)
\end{array}\right] \frac{t^{2}}{2}+\cdots \\
& C(t)=C_{0}+\left(\lambda D_{0}-\lambda \phi_{2} D_{0}-Q_{4} C_{0}\right) t \\
& +\left[\begin{array}{c}
\lambda\left(\alpha S_{0}-\alpha \phi_{1} S_{0}-Q_{3} D_{0}+\omega T_{0}\right) \\
-\lambda \phi_{2}\left(\alpha S_{0}-\alpha \phi_{1} S_{0}-Q_{3} D_{0}+\omega T_{0}\right) \\
-Q_{4}\left(\lambda D_{0}-\lambda \phi_{2} D_{0}-Q_{4} C_{0}\right)
\end{array}\right] \frac{t^{2}}{2}+\cdots \\
& T(t)=T_{0}+\left(\gamma C_{0}-Q_{5} T_{0}\right) t+\left[\begin{array}{c}
\gamma\left(\lambda D_{0}-\lambda \phi_{2} D_{0}-Q_{4} C_{0}\right) \\
-Q_{5}\left(\gamma C_{0}-Q_{5} T_{0}\right)
\end{array}\right] \frac{t^{2}}{2}+\cdots
\end{aligned}
$$




\subsection{Variables and estimation of parameter values}

Variables and parameter values were estimated based on the available data from [33], [25], [27], [38], [12], [3] and [2].

Table 2.3. Values of variables used for numerical simulation.

\begin{tabular}{|l|l|}
\hline Variables & Values \\
\hline$H(0)$ & $198,195,839$ \\
\hline$S(0)$ & $101,535,728$ \\
\hline$D(0)$ & 940,000 \\
\hline$C(0)$ & $3,760,000$ \\
\hline$T(0)$ & $1,193,250$ \\
\hline
\end{tabular}

Table 2.4. Values of parameters used for numerical simulation.

\begin{tabular}{|l|l|l|}
\hline Parameters & Values & Source \\
\hline$\alpha$ & 0.02 & {$[27]$} \\
\hline$\beta$ & 0.038 & {$[33]$} \\
\hline$\gamma$ & 0.08 & {$[10]$} \\
\hline$\lambda$ & 0.05 & {$[30]$} \\
\hline$\mu$ & 0.118 & {$[33]$} \\
\hline$\sigma$ & 0.08 & {$[7]$} \\
\hline$\theta$ & 0.923 & {$[31]$} \\
\hline$\phi_{1}$ & 0.5 & Assumed \\
\hline$\phi_{2}$ & 0.5 & Assumed \\
\hline$\omega$ & 0.08 & {$[30]$} \\
\hline$\delta$ & 0.02 & {$[7]$} \\
\hline$\tau$ & 0.04 & {$[30]$} \\
\hline $1-\theta$ & 0.077 & {$[31]$} \\
\hline
\end{tabular}




\section{Numerical Simulation and Result}

Numerical simulation of the results obtained in Section 2.3 was carried out using mathematical software (MAPLE 18) and the graphical profiles of the system responses are presented below.

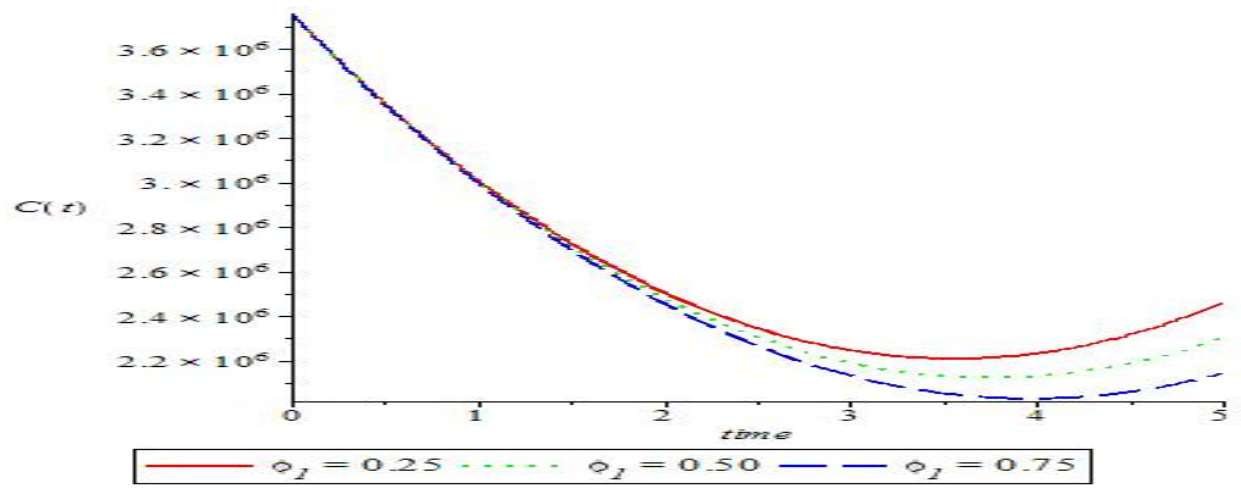

Figure 3.1. Effect of control measure $\phi_{1}$ on diabetics with complications class.

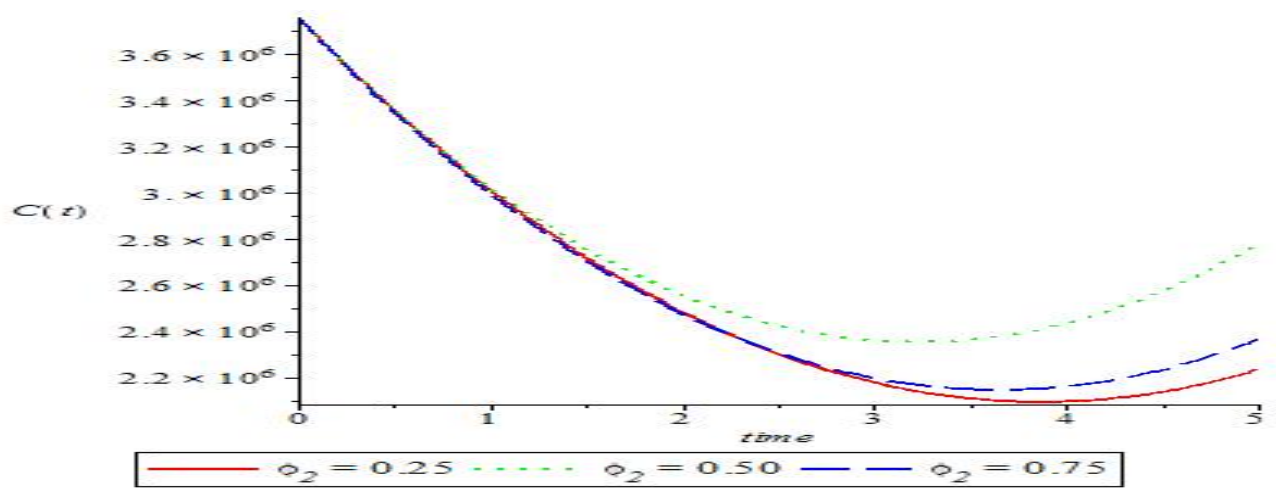

Figure 3.2. Effect of control measure $\phi_{2}$ on diabetics with complications class. 


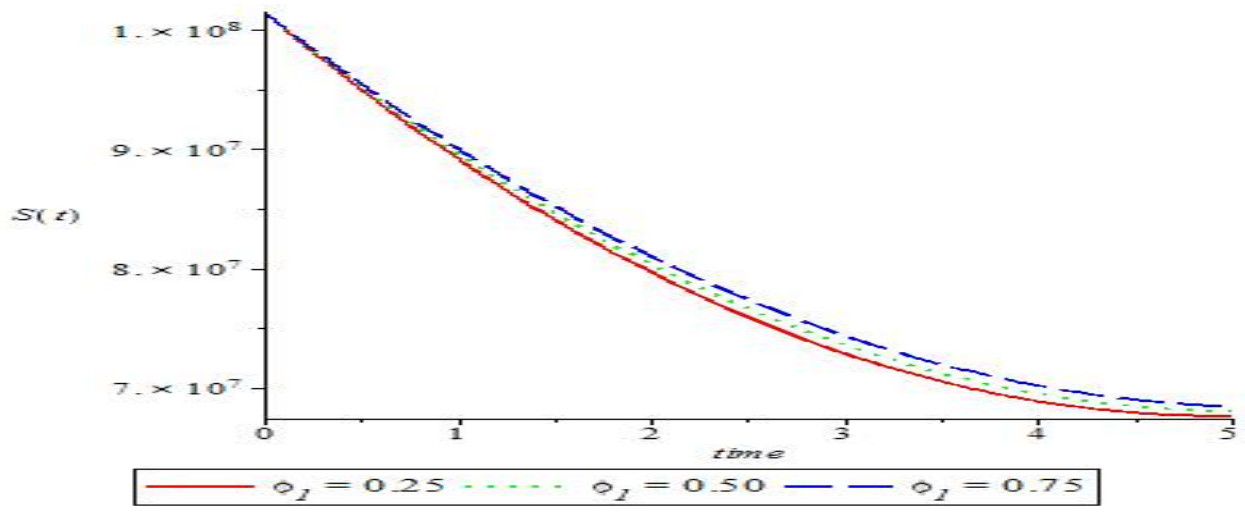

Figure 3.3. Effect of control measure $\phi_{1}$ on susceptible class.

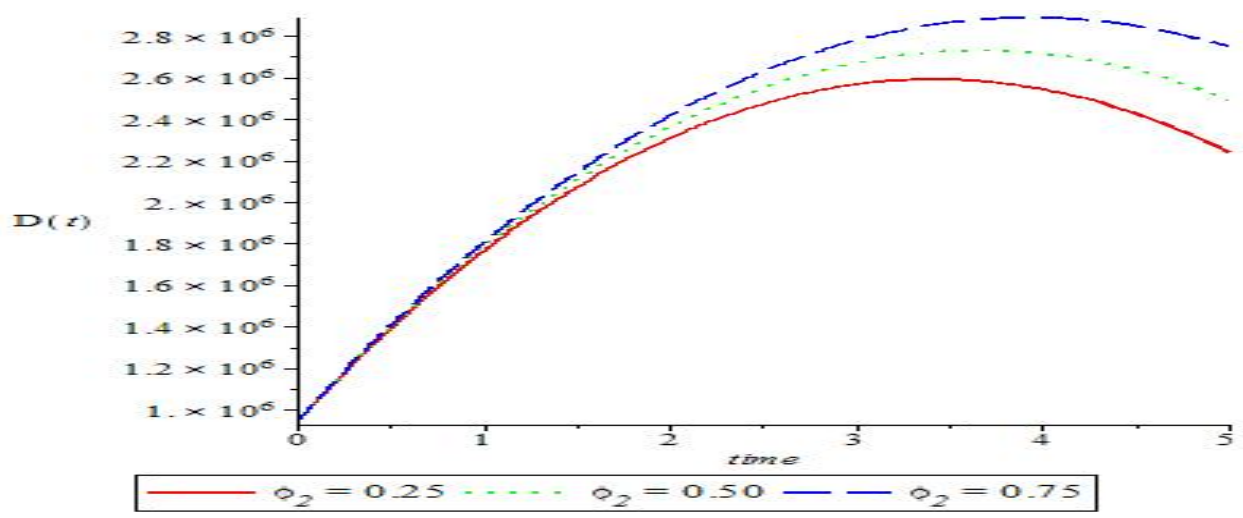

Figure 3.4. Effect of control measure $\phi_{2}$ on diabetics without complications class.

\section{Analysis of the Result}

Figure 3.1 shows that, as control rate increases, diabetics with complications decreases faster. This shows that the more people adopt positive lifestyle such as good dieting and regular physical exercises, the less cases of diabetics with complications. It is observed that as the control rate increases, the diabetics with complications is almost zero. However, effort to increase control measure will go a long way to reduce complications arising from diabetes. Figure 3.2 shows that, as control rate increases, diabetics with complications decreases. This shows that high rate of effective management of diabetes condition results to less cases of diabetics with complications. 
Figure 3.3 shows that, as control rate increases, susceptible class increases. This can be attributed to high rate of positive lifestyle. It pointed to the fact that the more people adopt positive lifestyle such as good dieting and regular physical exercises, the less they are susceptible to diabetes while Figure 3.4 shows that as control rate increases, diabetics without complications class increases. This is attributed to high rate of effective management of diabetes condition which helps in controlling the transition from diabetics without complications state to diabetics with complications state. It shows that the more effective management of diabetes condition, the less cases of complications.

\section{Conclusion}

The study presented a modified deterministic model for controlling the incidence of diabetes and its complications in a population. Two control parameters were imposed on the model equations and the effect of the control measures on the dynamics of diabetes are analysed. The model equations were solved analytically using homotopy perturbation method and solutions were obtained. The result showed that if control parameters rate could be increased, the transition rate from susceptible class to diabetics without complications class and transition rate from diabetics without complications to diabetics with complications would be drastically reduced. This will significantly reduce the incidence of diabetes and occurrence of complications in a population and the number of deaths attributable to diabetes and its complications would be minimised.

\section{References}

[1] N. Abubakar, N.I. Akinwande, O.R. Jimoh, F.A. Oguntolu and O.D. Ogwumu, Approximate solution of SIR infectious disease model using homotopy perturbation method (HPM), Pacific Journal of Science and Technology 14(2) (2013), 163-169.

[2] D. Adeloye, J.O. Ige, A.V. Aderemi, N. Adeleye, E.O. Amoo, A. Auta and G. Oni, Estimating the prevalence, hospitalization and mortality from type 2 diabetes mellitus in Nigeria, BMJ Open (2017). http://dx.doi.org/10.1136/bmjopen-2016-015424

[3] Africa Check, Does Nigeria have the most people with diabetes in Sub-Saharan Africa?, 2018. https://africacheck.org/fact-checks/reports/does-nigeria-have-most-peoplediabetes-sub-saharan-africa

[4] M.K. Ali, K.M. Bullard, E.W. Gregg and C.D. Rio, A cascade of care for diabetes in the United States: Visualizing the gaps, Annals of Internal Medicine 161(10) (2014), 681689. https://doi.org/10.7326/M14-0019 
[5] M.K. Ali, L.M. Jaacks, A.J. Kowalski, K.R. Siegel and M. Ezzati, Noncommunicable disease: three decades of global data show a mixture of increases and decreases in mortality rate, Health Affairs 34 (2015), 1444-1455.

https://doi.org/10.1377/hlthaff.2015.0570

[6] J. Biazar and H. Ghazvini, Exact solutions for non-linear Schrödinger equations by He's homotopy perturbation method, Phys. Lett. A 366 (2007), 79-84. https://doi.org/10.1016/j.physleta.2007.01.060

[7] A. Boutayeb, E.H. Twizell, K. Achouayb and A. Chetouani, A mathematical model for the burden of diabetes and its complications, BioMed. Eng. OnLine 3 (2004), Article No. 20. https://doi.org/10.1186/1475-925X-3-20

[8] M.S.H. Chowdhury and I. Hashim, Application of homotopy-perturbation method to Klein-Gordon and sine-Gordon equations, Chaos Solitons Fractals 39 (2009), 19281935. https://doi.org/10.1016/j.chaos.2007.06.091

[9] G. Danaei, C.M.M. Lawes, S.V. Hoorn and C.J.L. Murray and M. Ezzati, Global and regional mortality from ischaemic heart disease and stroke attributable to higher-thanoptimum blood glucose concentration: comparative risk assessment, The Lancet 368(9548) (2006), 1651-1659. https://doi.org/10.1016/S0140-6736(06)69700-6

[10] M. Derouich, A. Boutayeb, W. Boutayeb and M. Lamlili, Optimal control approach to the dynamics of a population of diabetes, Applied Mathematical Sciences 8(56) (2014), 2773-2782. https://doi.org/10.12988/ams.2014.43155

[11] K.E. Dooley and R.E. Chaisson, Tuberculosis and diabetes mellitus: convergence of two epidemics, The Lancet Infectious Diseases 9(12) (2009), 737-746.

https://doi.org/10.1016/S1473-3099(09)70282-8

[12] O.A. Fasanmade and S. Dagogo-Jack, Diabetes care in Nigeria, Annals of Global Health 81(6) (2016), 821-829. http://doi.org/10.1016/j.aogh.2015.12.012

[13] D.D. Ganji, and A. Rajabi, Assessment of homotopy-perturbation and perturbation methods in heat radiation equations, Int. Commun. Heat Mass Transfer 33 (2006), 391 400. https://doi.org/10.1016/j.icheatmasstransfer.2005.11.001

[14] D.D. Ganji, and A. Sadighi, Application of He's homotopy perturbation method to nonlinear coupled systems of reaction-diffusion equations, Int. J. Nonlinear Sci. Numer. Simul. 7(4) (2006), 411-418. https://doi.org/10.1515/IJNSNS.2006.7.4.411

[15] A.I. Enagi, M. Bawa and A.M. Sani, Mathematical study of diabetes and its complication using the homotopy perturbation method, International Journal of Mathematics and Computer Science 12(1) (2017), 43-63. 
[16] J.H. He, Homotopy perturbation technique, Computer Methods in Applied Mechanics and Engineering 178 (1999), 257-262. https://doi.org/10.1016/S0045-7825(99)00018-3

[17] J.H. He, A coupling method of homotopy technique and perturbation technique for nonlinear problems, Internat. J. Non-Linear Mech. 35 (2000a), 37-43. https://doi.org/10.1016/S0020-7462(98)00085-7

[18] J.H. He, New perturbation technique which is also valid for large parameters, J. Sound Vibration 229 (2000b), 1257-1263. http://dx.doi.org/10.1006/jsvi.1999.2509

[19] J.H. He, Asymptotology by homotopy perturbation method, Appl. Math. Comput. 156(3) (2004a), 591-596. https://doi.org/10.1016/j.amc.2003.08.011

[20] J.H. He, The homotopy perturbation method for nonlinear oscillators with discontinuities, Appl. Math. Comput. 151 (2004b), 287-292. https://doi.org/10.1016/S0096-3003(03)00341-2

[21] J.H. He, Application of homotopy perturbation method to nonlinear wave equations, Chaos Solitons Fractals 26 (2005a), 695-700. https://doi.org/10.1016/j.chaos.2005.03.006

[22] J.H. He, Homotopy perturbation method for bifurcation on nonlinear problems, Int. J. Non-linear Sci. Numer. Simul. 8 (2005b), 207-218.

[23] J.H. He, New interpretation of homotopy perturbation method, Internat. J. Modern Phys. B 20 (2006), 2561-2568. https://doi.org/10.1142/S0217979206034819

[24] International Diabetes Federation, IDF Diabetes Atlas, 6th Edition, 2013. http://www.idf.org/diabetesatlas

[25] International Diabetes Federation, About Diabetes, 2014. www.idf.org/about-diabetes,.

[26] International Diabetes Federation, IDF Diabetes Atlas, 7th Edition, Brussel: IDF, 2015. http://www.idf.org/diabetesatlas

[27] International Diabetes Federation, IDF Diabetes Atlas: Global Estimates of Diabetes Prevalence for 2017 and Projections for 2045, 2015.

[28] M.J. Magee, H.M. Blumberg and K.M. Venkat Narayan, Commentary: Co-occurrence of tuberculosis and diabetes: new paradigm of epidemiological transition, International Journal of Epidemiology 40(2) (2011), 428-431. https://doi.org/10.1093/ije/dyq268

[29] S. Odibat and S. Momani, A reliable treatment of homotopy perturbation method for Klein-Gordon equations, Phys. Lett. A 365 (2007), 351-357.

https://doi.org/10.1016/j.physleta.2007.01.064 
[30] A.H. Permatasari, R.H. Tjahjana and T. Udjiani, Global stability for linear system and controllability for nonlinear system in the dynamics model of diabetics population, $J$. Phys.: Conf. Ser. 1025 (2018), 012086.

https://doi.org/10.1088/1742-6596/1025/1/012086

[31] W. Purnami, C.A. Rifqi and R.S.S. Dewi, A mathematical model for the epidemiology of diabetes mellitus with lifestyle and genetic factors, J. Phys.: Conf. Ser. 1028 (2018), 012110. https://doi.org/10.1088/1742-6596/1028/1/012110

[32] G. Roglic, N. Unwin, P.H. Bennett, C. Mathers and J. Tuomilehto, The burden of mortality attributable to diabetes: realistic estimates for the year 2000, Diabetes Care 28(9) (2005), 2130-2135. https://doi.org/10.2337/diacare.28.9.2130

[33] United Nations, World Population Report, 2019. www.worldometers.info

[34] World Health Organization, 2008-2013, Action plan, for the global strategy for the prevention and control of non-communicable diseases, Geneva: WHO Document Services, 2008.

[35] World Health Organization, Department of Noncommunicable Disease Surveillance Geneva, Definition, Diagnosis and Classification of Diabetes Mellitus and its Complications, WHO/NCD/NCS/99.2, 1999.

[36] World Health Organization, Diabetes, Facts Sheet No. 312, 2014. Updated November 2014. www.who.org

[37] World Health Organization and International Diabetes Federation, Diabetic Action Now: An Initiative of the WHO and IDF, 2004. www.who.org or ww.idf.org/workshop

[38] World Health Organization, Diabetes Country Profile: Diabetes Prevalence in Some African Countries, 2016.

This is an open access article distributed under the terms of the Creative Commons Attribution License (http://creativecommons.org/licenses/by/4.0/), which permits unrestricted, use, distribution and reproduction in any medium, or format for any purpose, even commercially provided the work is properly cited. 